\title{
Anharmonicity and the isotope effect in superconducting lithium at high pressures: A first-principles approach
}

\author{
Miguel Borinaga, ${ }^{1,2}$ Unai Aseginolaza, ${ }^{1,2}$ Ion Errea, ${ }^{2,3}$ Matteo Calandra, ${ }^{4}$ Francesco Mauri, ${ }^{5}$ and Aitor Bergara ${ }^{1,2,6}$ \\ ${ }^{1}$ Centro de Física de Materiales CFM, CSIC-UPV/EHU, Paseo Manuel de Lardizabal 5, \\ 20018 Donostia/San Sebastián, Basque Country, Spain \\ ${ }^{2}$ Donostia International Physics Center (DIPC), Manuel Lardizabal pasealekua 4, \\ 20018 Donostia/San Sebastián, Basque Country, Spain \\ ${ }^{3}$ Fisika Aplikatua 1 Saila, Bilboko Ingeniaritza Eskola, University of the Basque Country (UPV/EHU), \\ Rafael Moreno "Pitxitxi” Pasealekua 3, 48013 Bilbao, Basque Country, Spain \\ ${ }^{4}$ IMPMC, UMR CNRS 7590, Sorbonne Universités-UPMC Univ. Paris 06, MNHN, IRD, 4 Place Jussieu, F-75005 Paris, France \\ ${ }^{5}$ Dipartimento di Fisica, Università di Roma La Sapienza, Piazzale Aldo Moro 5, I-00185 Roma, Italy \\ ${ }^{6}$ Departamento de Física de la Materia Condensada, University of the Basque Country (UPV/EHU), \\ 48080 Bilbao, Basque Country, Spain \\ (Received 16 March 2017; published 8 November 2017)
}

\begin{abstract}
Recent experiments [A. M. Schaeffer et al., Proc. Natl. Acad. Sci. USA 112, 60 (2015)] have shown that lithium presents an extremely anomalous isotope effect in the 15-25 GPa pressure range. In this article we have calculated the anharmonic phonon dispersion of ${ }^{7} \mathrm{Li}$ and ${ }^{6} \mathrm{Li}$ under pressure, their superconducting transition temperatures, and the associated isotope effect. We have found a huge anharmonic renormalization of a transverse acoustic soft mode along $\Gamma K$ in the fcc phase, the expected structure at the pressure range of interest. In fact, the anharmonic correction dynamically stabilizes the fcc phase above $25 \mathrm{GPa}$. However, we have not found any anomalous scaling of the superconducting temperature with the isotopic mass. Additionally, we have also analyzed whether the two lithium isotopes adopting different structures could explain the observed anomalous behavior. According to our enthalpy calculations including zero-point motion and anharmonicity it would not be possible in a stable regime.
\end{abstract}

DOI: 10.1103/PhysRevB.96.184505

\section{INTRODUCTION}

The strongly anomalous isotope effect recently measured in lithium in the 15-25 GPa pressure range [1] brought this element back under the spotlight. The reported superconducting critical temperatures $\left(T_{c}\right)$ contrast starkly with the BCS theory, where $T_{c}$ is expected to scale as $\propto 1 / M^{\alpha}$, with $M$ being the atomic mass and $\alpha$ the isotope coefficient ( 0.5 within the BCS theory). Actually, for most phonon mediated superconductors, $\alpha$ does not deviate much from 0.5. However, the above mentioned experiment shows a highly erratic behavior of $\alpha$ as a function of pressure, with values ranging from 1 to 4 from 15 to $21 \mathrm{GPa}$, decreasing sharply between 21 and $25 \mathrm{GPa}$, where it even becomes negative, with values as low as -2 .

It is just another fascinating example of the rich and exotic phenomena emerging in lithium under pressure. The lightest metal on the Periodic Table shows a nearly free-electron bcc structure at ambient conditions [2]. Although it could be expected to evolve to an even more free-electron-like system with increasing pressure, it has been shown that pressure not only induces several structural transformations [3-7], but also gives rise to a plethora of fascinating physical properties [8]. For instance, lithium becomes a semiconductor near $80 \mathrm{GPa}$ [9], it shows a maximum in the melting line [10], and melts below ambient temperature $(190 \mathrm{~K})$ at around $50 \mathrm{GPa}$ [3]. It also presents one of the highest $T_{c}$ for an element [1,11-15] and it is expected to display a periodic undamped plasmon $[16,17]$. Additionally, according to a recent experiment lithium shows quantum and isotope effects in its low temperature and pressure phase transformations [7].
Experimental evidence [3-7,9] shows that in the pressure and temperature ranges where the anomalous isotope effect was measured (15-25 GPa and below $30 \mathrm{~K}$ ) lithium presents a fcc structure. At around $40 \mathrm{GPa}$, it transforms to the rhombohedral hR1 phase, which is just a distortion of the fcc phase along the $c$ axis if one switches to a hexagonal representation. The transformation to the cubic cI16 phase occurs shortly after, at around $43 \mathrm{GPa}$.

Theoretical calculations within the harmonic approximation in fcc lithium show a highly softened transverse acoustic mode in the $\Gamma K$ high-symmetry line $[13,18-21]$. Around $\mathbf{q}_{\text {inst }}=2 \pi / a(2 / 3,2 / 3,0)$, where $a$ is the lattice parameter, this anomalous mode presents a huge electron-phonon coupling, becoming a key factor to explain the high $T_{c}$ observed in lithium [18-20]. This softening is associated to a well defined Fermi surface nesting [13,18-22] and even yields imaginary phonon frequencies at pressures where fcc is known to be stable; the instability emerges at pressures higher than $30 \mathrm{GPa}$ in the local density approximation (LDA), and at even lower pressures if one uses the generalized gradient approximation (GGA). As seen in other systems, such as simple cubic $\mathrm{Ca}$ [23], $\mathrm{PdH}$ [24], the record superconductor $\mathrm{H}_{3} \mathrm{~S}$ [25], and $\mathrm{NbSe}_{2}$ [26], anharmonicity is expected to have a significant role stabilizing this structure and, due to phonon frequency renormalization, also determining its superconducting properties [27]. As it has been measured at low pressures [7], zero-point vibrational energy could strongly impact the phase transitions of lithium in the 15-25 GPa pressure range, especially considering the small enthalpy differences between the most competitive candidates according to previous calculations $[4,28,29]$. In 
fact, the anharmonic correction to the vibrational energy could be significant as well.

The origin of the observed unconventional isotope effect in high pressure lithium remains unclear. Here we consider the following two hypotheses to explain this behavior. (i) Phonon frequencies scale with the atomic mass differently as expected within the harmonic approximation. Therefore, while in the harmonic approach the electron-phonon coupling constant $\lambda$ is independent of the isotopic mass, anharmonicity could make it differ from one isotope to the other, as it happens in palladium hydride [24]. (ii) ${ }^{6} \mathrm{Li}$ and ${ }^{7} \mathrm{Li}$ isotopes adopt different crystal structures due to the significant role of the vibrational energy in the phase diagram. Experimental evidence and previous theoretical calculations claim $\mathrm{Li}$ adopts the fcc phase from as low as $7 \mathrm{GPa}$ to as high as $40 \mathrm{GPa}$ in the temperature regime where superconductivity has been measured $[3,5,6,9]$. However, there is a considerable lack of experimental data in the mentioned region of the phase diagram and all previous calculations have been done in the static approach.

In this work we present an exhaustive analysis of the superconducting properties of fcc and cI16 structures of lithium in the 15-45 GPa pressure range, with vibrational degrees of freedom treated at the anharmonic level. We also analyze the possible existence of the $\mathrm{hR} 1$ phase in the pressure range of interest.

\section{COMPUTATIONAL DETAILS}

Our density functional theory (DFT) calculations were done within the Perdew-Burke-Ernzerhof (PBE) parametrization of the GGA [30]. Harmonic phonon frequencies and the electronphonon deformation potential were calculated within density functional perturbation theory (DFPT) [31] as implemented in QUANTUM ESPRESSO [32]. The electron-proton interaction was considered making use of an ultrasoft pseudopotential [33], which includes $1 s$ and $2 s$ electrons. Anharmonic calculations, including the vibrational contribution to the enthalpy, were performed using the stochastic self-consistent harmonic approximation (SSCHA) [34]. Anharmonic force constant matrices of fcc lithium were obtained by calculating forces in $3 \times 3 \times 3$ supercells. Therefore, anharmonic dynamical matrices were obtained in the respective commensurate $\mathbf{q}$-point grids and interpolated to a finer $9 \times 9 \times 9$ mesh afterwards. These were combined with DFPT electron-phonon calculations obtained in the fine $9 \times 9 \times 9$ mesh to calculate the anharmonic Eliashberg function $\alpha^{2} F(\omega)$. The same procedure was used for the cIl 6 structure, with $2 \times 2 \times 2$ and $6 \times 6 \times 6$ being the coarse and fine grids, respectively. The vibrational contribution to the enthalpy of hR1, which is a distortion of the fcc phase, was calculated using a $2 \times 2 \times 2$ grid for obtaining anharmonic force constant matrices and interpolating the differences with respect to the undistorted fcc structure. More details and the convergence parameters are given in the Supplemental Material [35].

\section{RESULTS AND DISCUSSION}

Figure 1 shows the DFPT harmonic phonon dispersion of fcc ${ }^{7} \mathrm{Li}$ at $26 \mathrm{GPa}$ and the anharmonic corrections calculated within the SSCHA. Anharmonic force constant matrices were

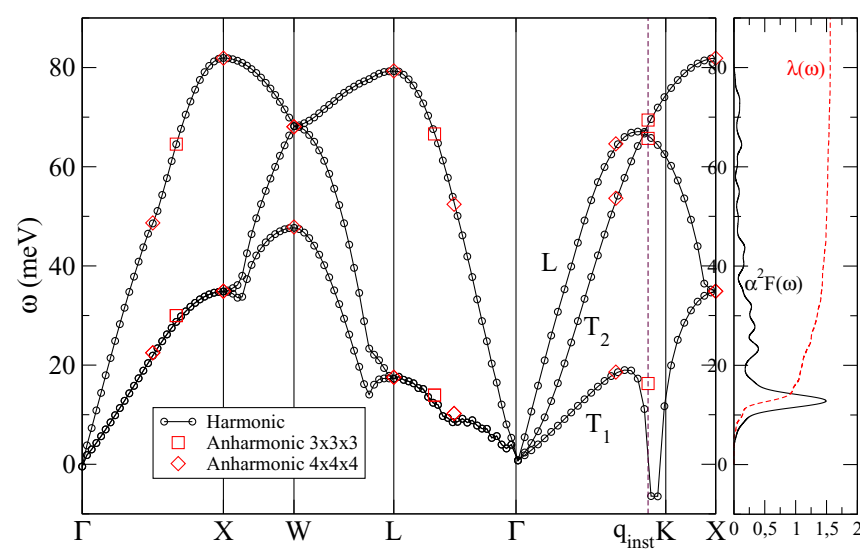

FIG. 1. Fcc ${ }^{7} \mathrm{Li}$ phonon dispersion at $26 \mathrm{GPa}$. Anharmonic phonons within the SSCHA are calculated both for a $3 \times 3 \times 3$ and a $4 \times 4 \times 4$ grid of points. The Eliashberg function $\alpha^{2} F(\omega)$ and the integrated electron-phonon coupling $\lambda(\omega)$ is also shown for the anharmonic case.

obtained by calculating forces in $3 \times 3 \times 3$ and $4 \times 4 \times 4$ supercells. Consequently, anharmonic dynamical matrices were obtained in the respective commensurate q-point grids. We see that anharmonicity is primarily localized around the phonon softening at the transverse acoustic $T_{1}$ branch at $\mathbf{q}_{\text {inst }}$, where the frequency is strongly shifted up by anharmonic effects. This well known phonon softening has been widely analyzed and explained in terms of Fermi surface nesting [13,18-22] and, as shown in Fig. 2, it even yields imaginary frequencies at pressures higher than $25 \mathrm{GPa}$, a considerably lower pressure than the $30 \mathrm{GPa}$ obtained within the LDA. In the same graph we also show the anharmonic frequency of the same mode, confirming fcc lithium is dynamically stabilized by anharmonicity above $25 \mathrm{GPa}$. However, as it is shown in the inset and even though this soft mode shows huge anharmonic effects, its frequency scales practically as in the harmonic case $(\omega \propto \sqrt{1 / M})$. Despite the large anharmonicity, a similar

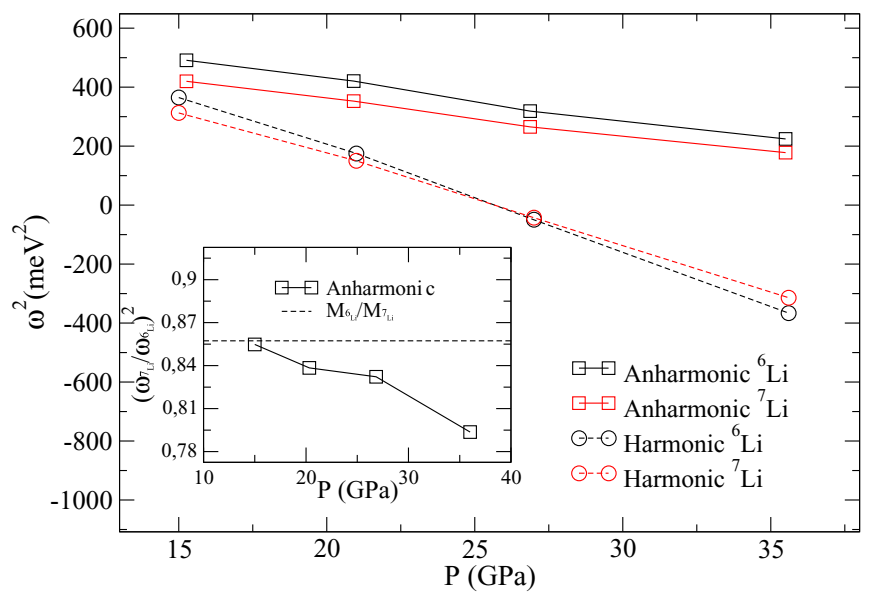

FIG. 2. Squared phonon frequencies of the anomalous transverse acoustic mode at $\mathbf{q}_{\text {inst }}$ for ${ }^{6} \mathrm{Li}$ and ${ }^{7} \mathrm{Li}$ isotopes as a function of pressure. The inset shows the ratio of the frequencies for both isotopes at the anharmonic level, with $M_{6}{ }_{\mathrm{Li}} / M_{7} \mathrm{Li}$ being the harmonic value. 


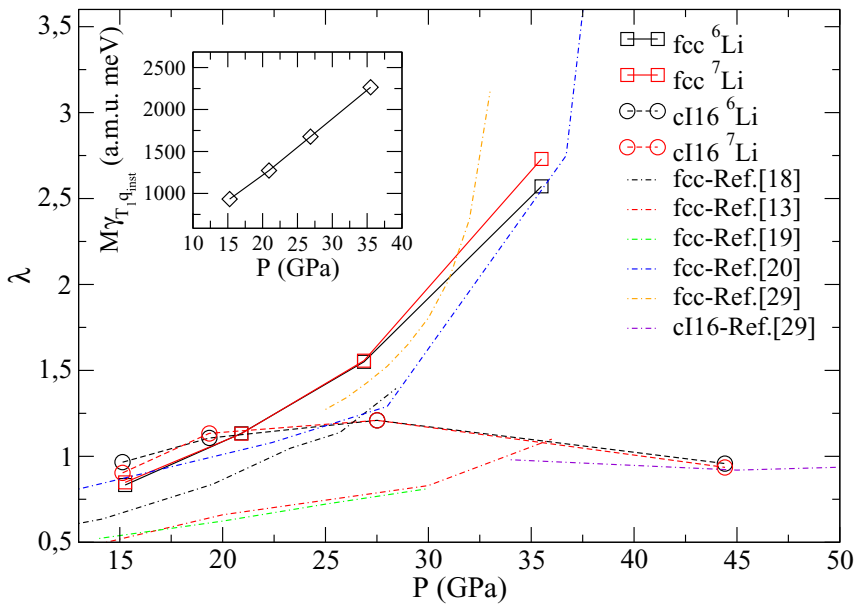

FIG. 3. Total electron-phonon coupling constant $\lambda$ of fcc and cI16 lithium calculated for its two isotopes at different pressures. The inset shows the phonon linewidth of the $T_{1}$ mode of fcc Li at $\mathbf{q}_{\text {inst }}$ multiplied by the atomic mass, the product being independent of the phonon frequency and the isotopic mass. The calculated $\lambda$ is compared to previous calculations [13,18-20,29].

harmonic scaling was previously calculated for high pressure simple cubic calcium [23].

Our DFPT electron-phonon coupling calculations displayed in Fig. 3 show the total coupling constant $\lambda$ rises abruptly with increasing pressure in the fcc phase. Starting from an already high value of 0.85 at $15 \mathrm{GPa}$ and reaching a value as high as 2.6 at $36 \mathrm{GPa}$, this dramatic growth is directly related to the also rapid increase of the electron-phonon linewidth $\gamma$ of the $T_{1}$ mode at $\mathbf{q}_{\text {inst }}$, which doubles its value in the mentioned pressure range. The remarkable peak in the Eliashberg function $\alpha^{2} F(\omega)$ and the associated abrupt growth of the integrated electron-phonon coupling constant $\lambda(\omega)$ around the frequency of the anomaly is another indicator of how relevant this softening is in the superconducting properties of fcc lithium. However, while the phonon renormalization of the mentioned mode due to anharmonicity is huge, $\lambda$ is nearly identical for both isotopes at every pressure except at $35 \mathrm{GPa}$, where the difference is just 7\%, even if anharmonicity is already really strong. As mentioned above, this is due to the fact that the frequency of the anomalous mode scales harmonically. Our $\lambda$ values are slightly larger than the ones by Maheswari et al. [20] and Profeta et al. [18] and quite larger than the ones by Akashi et al. [19] and Bazhirov et al. [13]. We attribute these disagreements to the large dependence of $\lambda$ with the q-point grid. While we used a $9 \times 9 \times 9$ sampling of the BZ for the electron-phonon and lattice dynamics calculations, where $\mathbf{q}_{\text {inst }}$ is explicitly taken into account, the mentioned works use $8 \times 8 \times 8$ grids $(7 \times 7 \times 7$ in the case of Maheswari et al.), where it is not. According to our convergence tests, those grids clearly underestimate $\lambda$ due to the absence of $\mathbf{q}_{\text {inst }}$ in the grid (see Supplemental Material [35]). Including this extremely anharmonic anomalous point is crucial for estimating the impact of anharmonicity in the electron-phonon coupling and, as a consequence, the superconducting $T_{c}$.

Considering that for large electron-phonon coupling constants the McMillan equation underestimates the supercon-

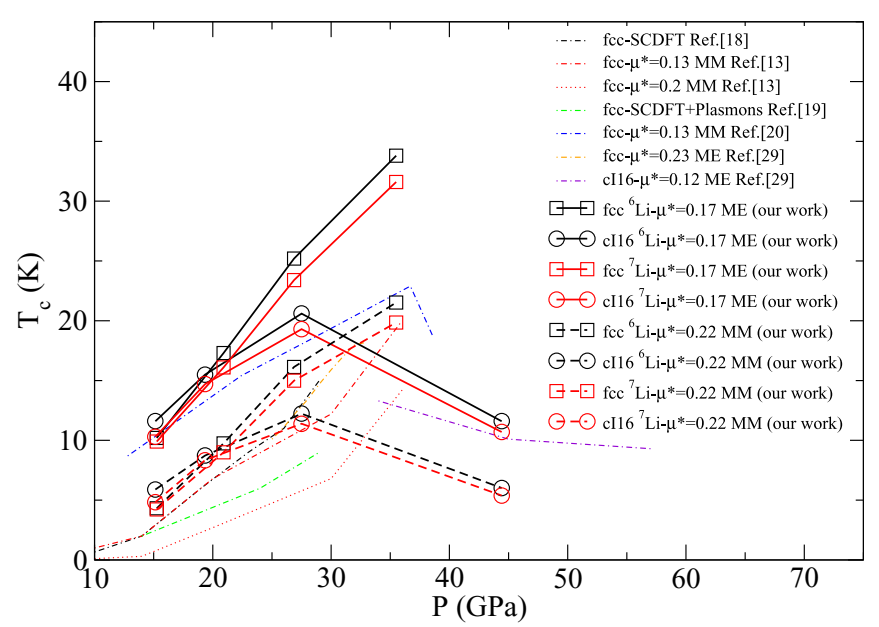

(a)

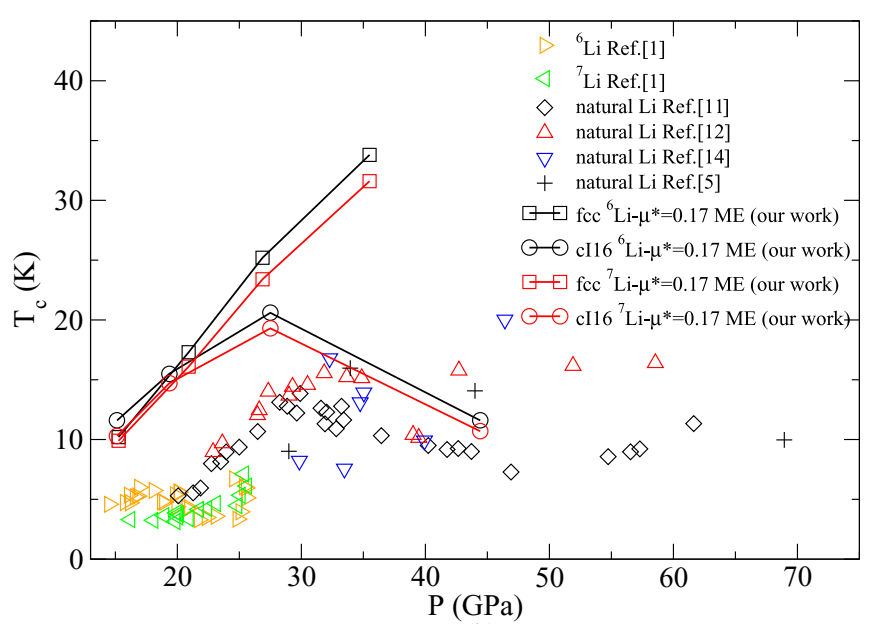

(b)

FIG. 4. $T_{c}$ estimations and comparison with (a) previous theoretical and (b) experimental results. (a) Estimated $T_{c}$ of fcc and cI16 lithium for its two stable isotopes at different pressures (lines with symbols) and comparison with other theoretical estimations (dashed and dotted curves) [13,18-20,29]. (b) Estimated $T_{c}$ of fcc and cI16 lithium for its two stable isotopes at different pressures (lines with symbols) and comparison with experimental values (characters) $[1,5,11,12,14] . T_{c}$ calculations are performed within McMillan (MM) and Migdal-Eliashberg (ME) approaches.

ducting $T_{c}$ [36], we solved the isotropic Migdal-Eliashberg equations $[37,38]$. We estimated a $\mu^{*}$ value of 0.17 using the Morel-Anderson formula [39]:

$$
\mu^{*}=\frac{\mu}{1+\ln \left(\frac{\varepsilon_{f}}{\omega_{D}}\right)} .
$$

The average electron-electron Coulomb repulsion term $\mu$ was obtained from Thomas-Fermi screening theory, a freeelectron Fermi energy $\varepsilon_{f}$ was chosen, and the Debye cutoff phonon frequency $\omega_{D}$ was taken as the highest frequency of the longitudinal acoustic modes [40]. Changes in phonon frequencies and electronic density for different pressures and isotopes only alter the fourth significant digit of $\mu^{*}$, so that differences in $\mu^{*}$ cannot explain the isotope effect anomalies and we assume the same value for both isotopes. Figure 4 


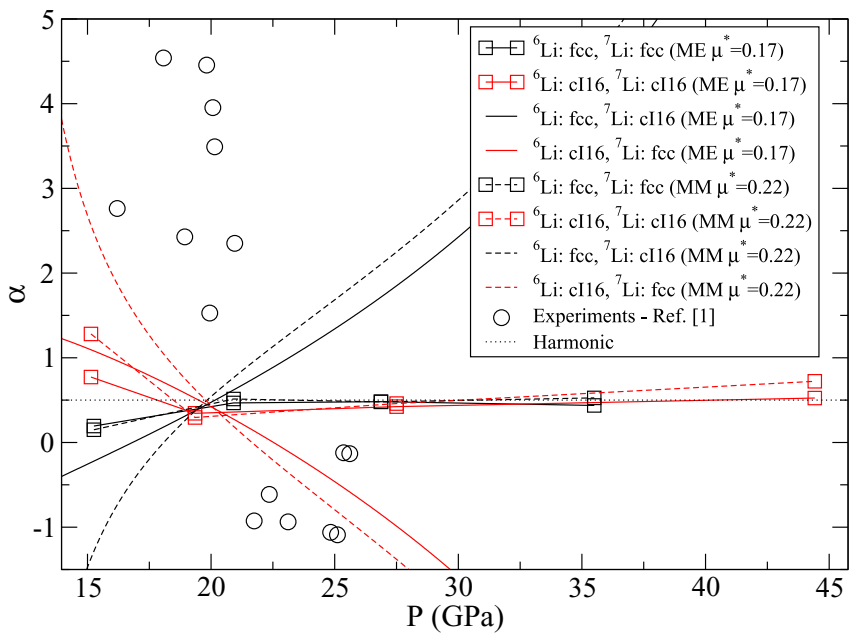

FIG. 5. Isotope coefficient $\alpha$ against pressure. Lines with symbols show the coefficients obtained for the cases in which the two isotopes adopt the same crystal structure (either cI16 or fcc). Curves without symbols show the coefficients for the cases in which the isotopes adopt different structures.

shows the superconducting critical temperature of fcc lithium for both isotopes at 15,20,26, and $36 \mathrm{GPa}$. We find $T_{c}$ increases monotonically with pressure the same way $\lambda$ does, ranging from $11.2 \mathrm{~K}(10.7 \mathrm{~K})$ at $15 \mathrm{GPa}$ to $34.8 \mathrm{~K}(32.5 \mathrm{~K})$ at $36 \mathrm{GPa}$ for ${ }^{6} \mathrm{Li}\left({ }^{7} \mathrm{Li}\right)$. As in the case of $\lambda$, we do not see any anomalous scaling of the superconducting temperature with the isotopic mass; as it can be seen in Fig. 5, $\alpha$ is close to the conventional harmonic $\mathrm{BCS}$ value of 0.5 within the entire pressure range except at $15 \mathrm{GPa}$ where, even though it shows a lower value, it does not, in any case, explain the experimentally observed anomalous isotope effect. Using McMillan's formula with $\mu^{*}=0.22 T_{c}$ compares better with literature and experiments, even though values are still larger than in previous works due to the choice of the q-point grid as in the case of $\lambda$; in any case, $\alpha$ does not almost change and the conclusion remains unaltered. The overestimation of $T_{c}$ could also indicate that vertex corrections in the electron-phonon coupling and anisotropic effects in the Migdal-Eliashberg equations might be important. However, anisotropic effects should not be isotope dependent and, due to the harmonic scaling of phonon frequencies, we do not expect vertex corrections to yield any anomalous isotope effect either. Therefore, we discard hypothesis (i).

After discarding that the anomalous isotope effect comes from strong anharmonicity in the fcc phase, we analyzed the possibility of the two isotopes showing different structures at the same pressure in a thermodynamically stable way. Figure 6 shows the enthalpies of the competing phases cI16 and hR1 relative to their respective fcc ones for the two isotopes. Our static calculations, i.e., not including zero-point energy (ZPE), compare well with literature (there are no previous works including ZPE) [28] and just show the fcc to cI16 transition. No important changes are shown for both isotopes when anharmonic ZPE is included and, although in the pressure range where this phase transition happens the enthalpy difference with the $\mathrm{hR} 1$ is less than $1 \mathrm{meV}$ per atom, that is, roughly the same as the error one assumes when converging

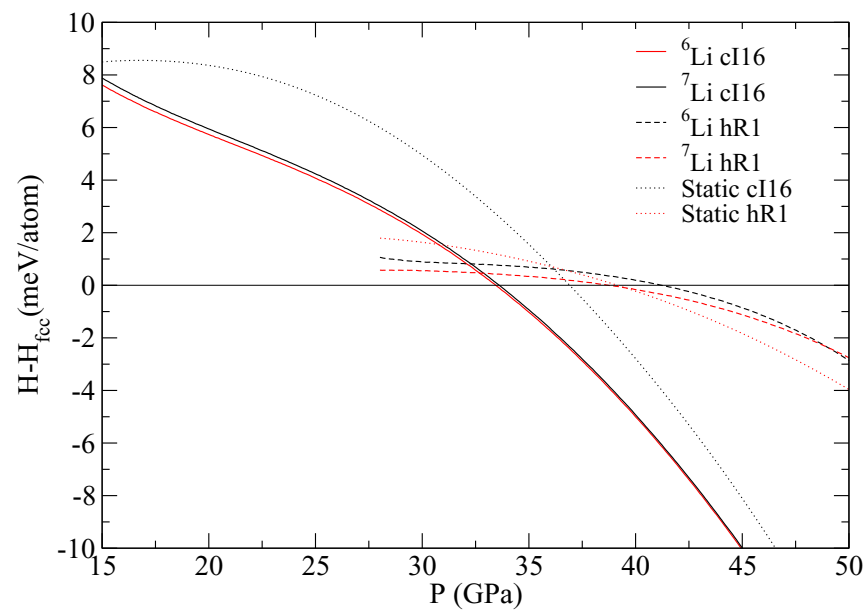

FIG. 6. Relative enthalpies of cI16 and $\mathrm{hR} 1{ }^{6} \mathrm{Li}$ and ${ }^{7} \mathrm{Li}$ isotopes with respect to their fcc counterparts. In solid and dashed lines ZPE has been included, while in dotted curves only electronic energy has been considered. The low pressure limit for the hR 1 curves has been set at the pressure which corresponds, in each case, to the maximum volume at which the phase shows a local minimum in the total energy surface (see Supplemental Material [35]).

total energy calculations within DFT, it remains metastable. Therefore, small changes in the calculation parameters or the choice of exchange and correlation potential might cause modifications in the transition pressures and phase sequence. Accordingly, when ZPE is included the fcc to cI16 transition pressure shifts from $37 \mathrm{GPa}$ to $33 \mathrm{GPa}$ for both isotopes, as the enthalpy difference is reduced by around $3 \mathrm{meV}$ due to lattice vibrations. Additionally, in the 21-25 GPa pressure range, where the inverse isotope effect was observed, the enthalpy difference between cI16 and fcc structures is really small (around 4-6 meV/atom). In conclusion, our results do not support hypothesis (ii) as ${ }^{6} \mathrm{Li}$ and ${ }^{7} \mathrm{Li}$ isotopes are not expected to adopt different stable crystal structures.

Due to the extremely small enthalpy differences metastable coexistence of phases cannot be discarded as it happens at ambient pressure for its martensitic transition [7]. In order to see if ${ }^{6} \mathrm{Li}$ and ${ }^{7} \mathrm{Li}$ adopting different structures could lead to the observed anomalous isotope effect, we have also made lattice dynamics and electron-phonon coupling calculations in the cI16 structure. We do not further consider hR1 as a candidate because, according to our calculations, the local minimum in the total energy surface associated to hR1 disappears for pressures lower than $28 \mathrm{GPa}$ (see Supplemental Material [35]). In Fig. 3 we show the total electron-phonon coupling $\lambda$ for cI16 $\mathrm{Li}$ at $15,19,27$, and $44 \mathrm{GPa}$. $\lambda$ does not vary with pressure as much as it does in the fcc phase; it varies only between 0.9 and 1.2 in the $15-44 \mathrm{GPa}$ pressure range. $\lambda$ is fairly similar for both isotopes, so that anharmonicity does not have almost any impact. Actually, at the lowest pressures, cI16 values differ more than the fcc ones from one isotope to the other. This is due to the fact that, while the overall phonon spectrum is very slightly modified by anharmonicity in the cI16 phase, anharmonic corrections occur mostly at the lowest frequencies, which are the ones that contribute most to the total electron-phonon coupling. In fact, our $\lambda$ and $T_{c}$ estimations, 
with $\mu^{*}=0.17$ obtained with the Morel-Anderson formula as in the fcc case, shown in Figs. 3 and 4 yield values higher than in fcc below $20 \mathrm{GPa}$, being the opposite at higher pressures. The isotope effect coefficient is close to the harmonic value at 27 and $44 \mathrm{GPa}$, with $\alpha=0.42$ and 0.57 , respectively, while it deviates considerably from 0.5 at 15 and $19 \mathrm{GPa}$ as it yields $\alpha=0.77$ and 0.34 , respectively. All this agrees with the higher anharmonicity we found at lower pressures. Although our enthalpy calculations do not predict both isotopes can stabilize in different structures, we have also analyzed this metastability driven hypothetical scenario: ${ }^{6} \mathrm{Li}$ stabilizing in the fcc phase and ${ }^{7} \mathrm{Li}$ in the cI16, and vice versa. As shown in Fig. 5, in the pressure range where the inverse isotope effect was experimentally observed (21-25 GPa), experimental values would only be qualitatively reproduced if ${ }^{6} \mathrm{Li}$ adopted the cI16 structure while ${ }^{7} \mathrm{Li}$ were in the fcc phase. This qualitative picture does not vary much if one uses the McMillan formula with $\mu^{*}=0.22$, but it could notably change if we used different $\mu^{*}$ values for the different phases.

\section{CONCLUSIONS}

According to our calculations, even though anharmonicity is crucial to stabilize the fcc phase in lithium under pressure, its $\lambda$ remains almost the same for both isotopes and yields a conventional scaling of $T_{c}$ with isotopic mass and, therefore, it does not explain the experimentally observed anomalous isotope effect. On the other hand, including anharmonic ZPE in the enthalpy curve does not modify lithium phase diagram in the pressure range of interest, so that it is unexpected to have both isotopes in different structures. The anomalous isotope effect could only be qualitatively explained if ${ }^{7} \mathrm{Li}$ adopted the fcc structure while ${ }^{6} \mathrm{Li}$ adopted the cI16 one in a metastable way. All this, added to the large error bars and quite chaotic behavior of $T_{c}$ with pressure in Ref. [1]—with considerably different temperature values for the same pressure, puts in question the experimental observation of an anomalous isotope effect in lithium at high pressure. This way, our work encourages further research to determine the phase sequence and superconducting properties of the two stable isotopes of lithium.

\section{ACKNOWLEDGMENTS}

The authors acknowledge financial support from the Spanish Ministry of Economy and Competitiveness (Grant No. FIS2016-76617-P) and the Department of Education, Universities and Research of the Basque Government and the University of the Basque Country (Grant No. IT756-13). M.B. is also thankful to the Department of Education, Language Policy and Culture of the Basque Government for a predoctoral fellowship (Grant No. PRE-2015-2-0269). Computer facilities were provided by PRACE and the Donostia International Physics Center (DIPC).
[1] A. M. Schaeffer, S. R. Temple, J. K. Bishop, and S. Deemyad, Proc. Natl. Acad. Sci. USA 112, 60 (2015).

[2] F. Seitz, Phys. Rev. 47, 400 (1935).

[3] C. L. Guillaume, E. Gregoryanz, O. Degtyareva, M. I. McMahon, M. Hanfland, S. Evans, M. Guthrie, S. V. Sinogeikin, and H.-K. Mao, Nat. Phys. 7, 211 (2011).

[4] M. Hanfland, K. Syassen, N. E. Christensen, and D. L. Novikov, Nature (London) 408, 174 (2000).

[5] T. Matsuoka, S. Onoda, M. Kaneshige, Y. Nakamoto, K. Shimizu, T. Kagayama, and Y. Ohishi, J. Phys.: Conf. Ser. 121, 052003 (2008).

[6] A. M. Schaeffer, W. Cai, E. Olejnik, J. J. Molaison, S. Sinogeikin, A. M. dos Santos, and S. Deemyad, Nat. Commun. 6, 8030 (2015).

[7] G. J. Ackland, M. Dunuwille, M. Martinez-Canales, I. Loa, R. Zhang, S. Sinogeikin, W. Cai, and S. Deemyad, Science 356, 1254 (2017).

[8] B. Rousseau, Y. Xie, Y. Ma, and A. Bergara, Eur. Phys. J. B 81, 1 (2011).

[9] T. Matsuoka and K. Shimizu, Nature (London) 458, 186 (2009).

[10] E. R. Hernández, A. Rodriguez-Prieto, A. Bergara, and D. Alfè, Phys. Rev. Lett. 104, 185701 (2010).

[11] S. Deemyad and J. S. Schilling, Phys. Rev. Lett. 91, 167001 (2003).

[12] V. V. Struzhkin, M. I. Eremets, W. Gan, H.-k. Mao, and R. J. Hemley, Science 298, 1213 (2002).

[13] T. Bazhirov, J. Noffsinger, and M. L. Cohen, Phys. Rev. B 82, 184509 (2010).

[14] K. Shimizu, H. Ishikawa, D. Takao, T. Yagi, and K. Amaya, Nature (London) 419, 597 (2002).
[15] N. W. Ashcroft, Nature (London) 419, 569 (2002).

[16] V. M. Silkin, A. Rodriguez-Prieto, A. Bergara, E. V. Chulkov, and P. M. Echenique, Phys. Rev. B 75, 172102 (2007).

[17] I. Errea, A. Rodriguez-Prieto, B. Rousseau, V. M. Silkin, and A. Bergara, Phys. Rev. B 81, 205105 (2010).

[18] G. Profeta, C. Franchini, N. N. Lathiotakis, A. Floris, A. Sanna, M. A. L. Marques, M. Lüders, S. Massidda, E. K. U. Gross, and A. Continenza, Phys. Rev. Lett. 96, 047003 (2006).

[19] R. Akashi and R. Arita, Phys. Rev. Lett. 111, 057006 (2013).

[20] S. U. Maheswari, H. Nagara, K. Kusakabe, and N. Suzuki, J. Phys. Soc. Jpn. 74, 3227 (2005).

[21] A. Rodriguez-Prieto, A. Bergara, V. M. Silkin, and P. M. Echenique, Phys. Rev. B 74, 172104 (2006).

[22] A. Rodriguez-Prieto and A. Bergara, Phys. Rev. B 72, 125406 (2005).

[23] I. Errea, B. Rousseau, and A. Bergara, J. Appl. Phys. 111, 112604 (2012).

[24] I. Errea, M. Calandra, and F. Mauri, Phys. Rev. Lett. 111, 177002 (2013).

[25] I. Errea, M. Calandra, C. J. Pickard, J. R. Nelson, R. J. Needs, Y. Li, H. Liu, Y. Zhang, Y. Ma, and F. Mauri, Nature (London) 532, 81 (2016).

[26] M. Leroux, I. Errea, M. Le Tacon, S.-M. Souliou, G. Garbarino, L. Cario, A. Bosak, F. Mauri, M. Calandra, and P. Rodière, Phys. Rev. B 92, 140303 (2015).

[27] M. Borinaga, U. Aseginolaza, I. Errea, A. Bergara, U. Aseginolaza, I. Errea, and A. Bergara, JJAP Conf. Proc. 6, 011103 (2017).

[28] Y. Ma, A. R. Oganov, and Y. Xie, Phys. Rev. B 78, 014102 (2008). 
[29] Y. Yao, J. S. Tse, K. Tanaka, F. Marsiglio, and Y. Ma, Phys. Rev. B 79, 054524 (2009).

[30] J. P. Perdew, K. Burke, and M. Ernzerhof, Phys. Rev. Lett. 77, 3865 (1996).

[31] S. Baroni, S. de Gironcoli, A. Dal Corso, and P. Giannozzi, Rev. Mod. Phys. 73, 515 (2001)

[32] P. Giannozzi, J. Phys.: Condens. Matter 21, 395502 (2009).

[33] D. Vanderbilt, Phys. Rev. B 41, 7892 (1990).

[34] I. Errea, M. Calandra, and F. Mauri, Phys. Rev. B 89, 064302 (2014).

[35] See Supplemental Material at http://link.aps.org/supplemental/ 10.1103/PhysRevB.96.184505 for more details on the conver- gence parameters of electronic, vibrational, and electron-phonon coupling calculations and on the interpolation methods of the enthalpy calculations.

[36] P. B. Allen and R. C. Dynes, Phys. Rev. B 12, 905 (1975).

[37] A. B. Migdal, Zh. Eksp. Teor. Fiz. 34, 1438 (1958) [Sov. Phys. JETP 7, 996 (1958)].

[38] G. M. Eliashberg, Zh. Eksp. Teor. Fiz. 38, 966 (1960) [Sov. Phys. JETP 11, 696 (1960)].

[39] P. Morel and P. W. Anderson, Phys. Rev. 125, 1263 (1962).

[40] K. J. Chang, M. M. Dacorogna, M. L. Cohen, J. M. Mignot, G. Chouteau, and G. Martinez, Phys. Rev. Lett. 54, 2375 (1985). 